\title{
无氧条件下 $\mathrm{Pt} / \mathrm{TiO}_{2}$ 光催化重整降解一乙醇胺水溶液制氢 *
}

\author{
吴玉琪吕功煊李树本 \\ (中国科学院兰州化学物理研究所, 羰基合成与选择氧化国家重点实验室, 兰州 730000 )
}

\begin{abstract}
摘要 以一乙醇胺 (以下简称乙醇胺) 为电子给体, 在无氧条件下进行了 $\mathrm{Pt} / \mathrm{TiO}_{2}$ 光催化重整制氢的研究. 详细 讨论了诸多因素如催化剂表面 $\mathrm{Pt}$ 化学状态、 $\mathrm{Pt}$ 担载量、溶液 $\mathrm{pH}$ 值、乙醇胺溶液浓度等对产氢效率的影响, 并用 $\mathrm{XRD} 、 \mathrm{HNMR} 、 \mathrm{XPS}$ 等进行了深人表征, 探讨了 $\mathrm{Pt} / \mathrm{TiO}_{2}$ 光催化重整降解乙醇胺和产氢的反应, 实验表明, 利用所 制备的光催化剂, 可实现在消除水中有机污染物的同时制取氢气的目标. 催化剂表面的 $\mathrm{Pt}$ 以 $\mathrm{Pt}^{0}$ 的化学状态存 在, 有利于析氢; 溶液 $\mathrm{pH}$ 值和浓度的变化对产生速率也有一定的影响. 同时发现 $\mathrm{Pt} / \mathrm{TiO}_{2}$ 光催化重整乙醇胺制 氢反应的最佳条件是: $\mathrm{Pt}$ 的最佳担载量约为 $0.5 \% \sim 1.0 \%$; 乙醇胺溶液最佳浓度约为 $0.05 \mathrm{~mol} \cdot \mathrm{L}^{-1}$; 最佳溶液 $\mathrm{pH}$ 值范围为 $4 \sim 10$; 氨基取代的羰基类化合物是其主要中间产物.
\end{abstract}

关键词: 光催化重整, 降解, 乙醇胺, $\mathrm{Pt} / \mathrm{TiO}_{2}$, 制氢 中图分类号: 0643.32

乙醇胺不仅是一种用途很广的有机物, 而且广 泛地用于医药、农药、表面活性剂、空气净化剂、化 工助剂、化妆品、化学武器、以及有机合成中间体等 许多精细化工产品的生产 ${ }^{[1-2]}$. 但是, 乙醇胺也是有 一定毒性的化合物 ${ }^{[3]}$. 如何消除生产过程排放物中 含有的少量乙醇胺污染物值得关注.光催化降解 水中有机污染物是目前国内外十分活跃的研究领 域 ${ }^{[4-8]}$. 发展半导体催化剂直接降解乙醇胺同时产氢 不仅对将来的分离工艺有重要意义, 而且对推进利 用三乙醇胺制氢影响深远. 对于无氧条件下乙醇 胺降解规律的认识也有帮助.

氢能是未来的最理想能源之一，而利用太阳能 光催化制氢技术是具吸引力和最具挑战性的. 本 文利用光催化剂实现了光催化重整氧化降解有毒 的乙醇胺的同时, 产生清洁无污染的燃料一一氢 气, 并详细讨论了诸多因素如催化剂表面化学状 态、活性组份的担载量、溶液 $\mathrm{pH}$ 值、乙醇胺溶液浓 度等对产氢速率的影响, 并探讨乙醇胺降解和产氢 的可能机理.

\section{1 实验部分}

\subsection{Pt 担载的 $\mathrm{TiO}_{2}$ 光催化剂的制备 ${ }^{[9-11]}$} 选用 P25 二氧化钛德国 Degussa 公司, 有效表
面积 $50 \mathrm{~m}^{2} \cdot \mathrm{g}^{-1}, 70 \%$ 锐钛矿, 粒径 $30 \mathrm{~nm}$ )为载体, 并根据 $\mathrm{Pt}$ 担载量的不同, 取一定量的氯铂酸 $\left(\mathrm{H}_{2} \mathrm{PtCl}_{6} \cdot 6 \mathrm{H}_{2} \mathrm{O}\right.$, 分析纯) 溶液与称量好的 $\mathrm{P} 25 \mathrm{TiO}_{2}$ 粉末混合, 加水稀释至 $80 \mathrm{~mL}$, 在 $200 \mathrm{~W}$ 弧光灯的 照射下用磁力搅拌器搅拌 $10 \mathrm{~h}$ 以上经过洗涤、离 心, 在 $110{ }^{\circ} \mathrm{C}$ 下干燥 $12 \mathrm{~h}$ 以上, 研磨成直径小于 $0.05 \mathrm{~mm}$ 的粉末待用.

\section{2 实验装置和产氢量的评估}

实验在石英反应器 ${ }^{[9-10]}$ 中进行。该反应器配有 隔绝空气的胶膜和一个温度计插孔, 以便取样和观 察反应温度变化. 将 $20 \mathrm{mg} \mathrm{Pt} / \mathrm{TiO}_{2}$ 催化剂和 80 $\mathrm{mL}$ 一定浓度的乙醇胺水溶液放人烧瓶中, 用氩气 置换 $40 \mathrm{~min}$ 以除去溶解在蒸馏水中的空气。用磁 力摚拌器摚拌, 使催化剂保持悬浮, $200 \mathrm{~W}$ 高压永 灯作为紫外光光源, 从反应器侧面照射, 每隔 15 $\min$ 抽取 $0.5 \mathrm{~mL}$ 气相样品, 在 GC-8800气相色谱 工作站上分析产氢量.

\section{3 光催化反应效率的评价方法}

由于本实验中光照反应时间相同 除特别说明 外均为 $120 \mathrm{~min}$ ）, 用相同时间内产生氢气的体积的 多少来评价反应效率.

\section{$1.41 .0 \% \mathrm{Pt} / \mathrm{TiO}_{2}$ 光催化剂 XPS 和 XRD 表征}

1. $0 \%(w) \mathrm{Pt} / \mathrm{TiO}_{2}$ 光催化剂的 XPS 用 ESCAL-

2003-12-16 收到初稿, 2004-2-16 收到修改稿。联系人: 吕功煊 (E-mail: gxlu @ ns. lzb. ac. cn; Tel: 0931-4968178). *国家重点基础研 究专项经费(2003CB214500)、国家自然科学基金(90210027)和国家基础研究重大项目计划 (G200000264)资助项目 


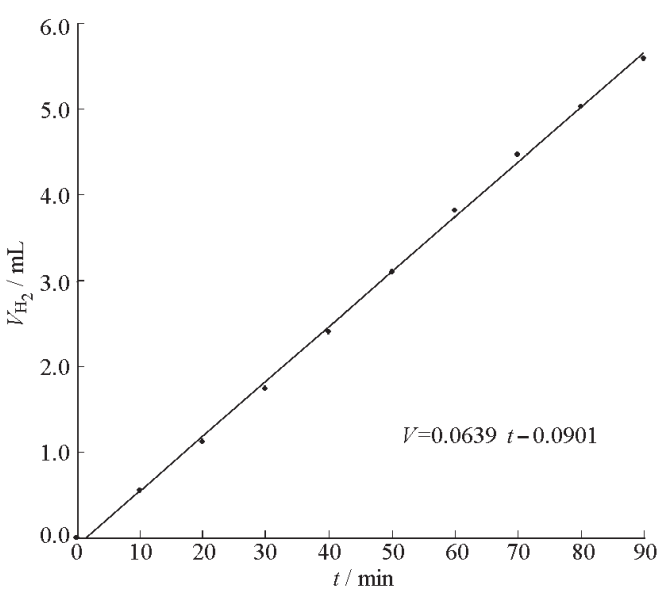

图 $11.0 \%(w)$ Pt- $\mathrm{TiO}_{2}$ 光催化重整乙醇胺溶液制 氢反应特征曲线

Fig. 1 The characteristic curve of photocatalytic reaction of aqueous ethanolamine solution using $1.0 \%(w) \mathrm{Pt} / \mathrm{TiO}_{2}$ photocatalyst

$\mathrm{AB} 210 \mathrm{XPS}$ 电子能谱仪, 采用 $\mathrm{Mg}$ 靶, 通能为 $30 \mathrm{eV}$, 步径为 $80 \mathrm{meV}, \mathrm{C} 1 s=284.6 \mathrm{eV}$ 校正的条 件下得到的。XRD 用 $\mathrm{D} / \max \mathrm{RB} X$ 射线衍射仪在 采用 $\mathrm{Cu}$ 靶，管压为 $50 \mathrm{kV}$, 管流为 $80 \mathrm{~mA}$, 扫描速 度为 $4\left(^{\circ}\right) \cdot \mathrm{min}^{-1}$, 步宽为 $0.01^{\circ}$ 的条件下得到的.

\section{5 光催化重整降解氯乙醇/重水溶液中间产物}

的 HNMR 谱图

光催化反应中间产物的 HNMR 谱图采用 Mercury plus 300BB NMR Spectrameter 表征.

\section{2 结果与讨论}

\section{$2.1 \mathrm{Pt}$ 担载 $\mathrm{TiO}_{2}$ 光催化重整制氢反应特征曲线}

图 1 是 $1.0 \%(w) \mathrm{Pt}$ 担载的 $\mathrm{TiO}_{2}$ 光催化重整 $0.05 \mathrm{~mol} \cdot \mathrm{L}^{-1}$ 乙醇胺溶液制氢反应的特征曲线.

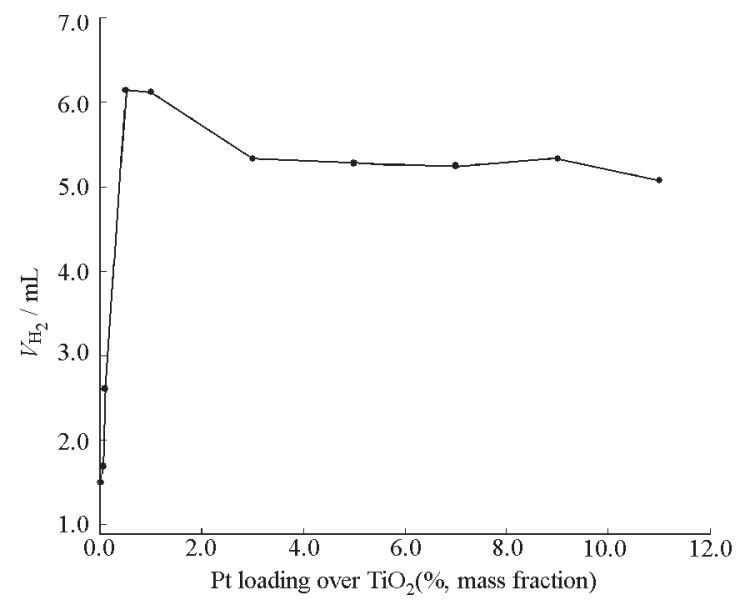

图 $2 P t$ 担载量对产氢效率的影响

Fig. 2 The effect of $\mathrm{Pt}$ loading on the rate of hydrogen production
从图中可以看到，产生氢气的体积与光照时间有很 好的线性关系, 说明在实验观察的范围内, 催化剂 性能未见下降, 放氢速率 $\left.6.0639 \mathrm{~mL} \cdot \mathrm{min}^{-1}\right)$ 略低 于乙醇溶液反应速率 ${ }^{[9-10]}$, 比光催化分解纯水的反 应速率 $\left(0.0026 \mathrm{~mL} \cdot \mathrm{min}^{-1}\right)$ 高很多。

\section{2 催化剂表面 Pt 化学状态}

催化剂表面的化学状态对光催化反应效率有明 显的影响 ${ }^{[9]}$. XRD 结果表明, 在 $2 \theta=25.24 、 48.12$ 、 37.84 和 $2 \theta=27.40 、 54.32 、 36.06$ 处有明显的、尖 锐的锐钛矿型和金红石型特征峰出现，说明在光沉 积过程中, $\mathrm{TiO}_{2}$ (P25)的晶体结构保持完整; XPS 分 析结果是 $E_{\left(\mathrm{P} t f_{\mathrm{J} / 2}\right)}=74.30 \mathrm{eV}, E_{\left(\mathrm{P} t 4 f_{/ 2}\right)}=70.87 \mathrm{eV}$, 对比 $\mathrm{Pt}$ 元素的结合能 (标准值为 $E_{\left(\mathrm{P} 4 f_{f_{2}}\right)}=74.25$ $\left.\mathrm{eV}, E_{\left(\mathrm{P} 4 f_{/ 2}\right)}=70.90 \mathrm{eV}\right)$ 说明分散于 $\mathrm{TiO}_{2}(\mathrm{P} 25)$ 表 面的 $\mathrm{Pt}$ 晶粒是以 $\mathrm{Pt}^{0}$ 价态存在. 当催化剂受到光照 时，在半导体 $\mathrm{TiO}_{2}$ 粒子表面形成的能垒能够有效 捕获光生电子, 大大降低了光生电子和空穴的复合 速率, 从而提高光催化反应效率.

\section{$2.3 \mathrm{Pt}$ 担载量对光催化反应效率的影响}

如图 2 所示, Pt 担载量对产氧效率有明显影响. 在较低担载量区, 产氧效率随着担载量的增加而增 加, 当担载量达到 $0.5 \%$ 时呈现最大值, 而后产氧效 率随担载量的增加略有下降. 这与 $\mathrm{Pt} / \mathrm{TiO}_{2}$ 光催化 重整降解氯乙醇的情况相似 ${ }^{[12]}$, 这种情况的出现可 能与底物有机分子自身性质有关。考虑到催化剂 生产成本和产氢效率, 最佳担载量为 $0.5 \%(w)$.

\section{4 乙醇胺浓度对光催化反应效率的影响}

乙醇胺起始浓度对产氢量也有很明显的影响 (如图 3 所示) 。在较低浓度区域（0.00 0.05 $\mathrm{mol} \cdot \mathrm{L}^{-1}$, 产氢量随着反应物乙醇胺的浓度的增加

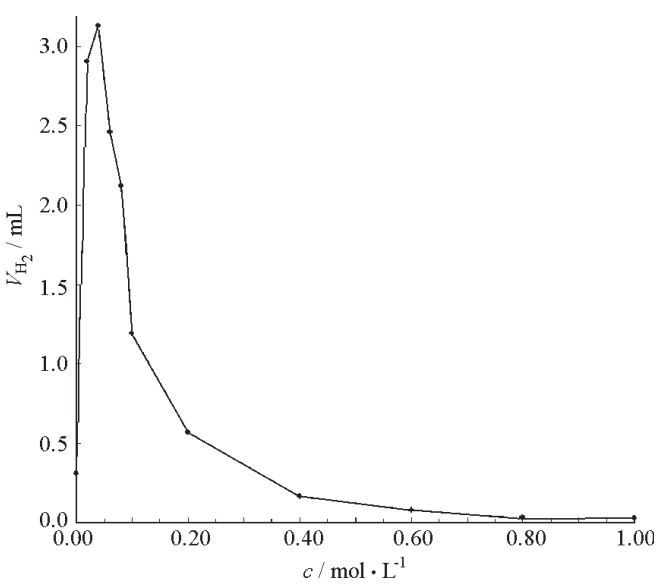

图 3 乙醇胺起始浓度 $(c)$ 对产氢量的影响

Fig. 3 The effect of initial ethanolamine concentration (c) on $\mathbf{H}_{2}$ production rate 


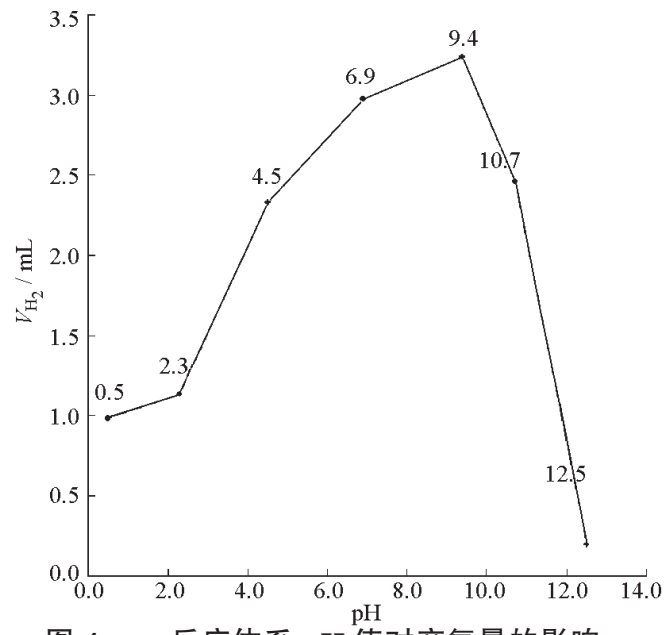

图 4 反应体系 pH 值对产氢量的影响

Fig. 4 The effect of $\mathrm{pH}$ value on hydrogen production rate

有明显增加, 并在 $0.05 \mathrm{~mol} \cdot \mathrm{L}^{-1}$ 左右达到最大值; 当乙醇胺浓度大于 $0.05 \mathrm{~mol} \cdot \mathrm{L}^{-1}$ 时，乙醇胺浓度 增加导致产氢量逐渐下降，当乙醇胺的浓度高于 $0.50 \mathrm{~mol} \cdot \mathrm{L}^{-1}$ 左右时产氢量下降到较低. 影响光 催化反应效率的因素是多方面的。在低浓度下，催 化剂表面吸附层乙醇胺浓度将会随着溶液体系浓度 的增加而增大，当吸附层浓度达到一个稳定的饱和 值时不再受溶液体系浓度增加的影响。乙醇胺浓度 继续增加时反应效率略有下降，是由于乙醇胺水溶 液因水解作用而呈碱性 朱经调节的 $0.05 \mathrm{~mol} \cdot \mathrm{L}^{-1}$ 乙醇胺水溶液 $\mathrm{pH}=10.70$ ), 溶液浓度的增加也会 改变溶液 $\mathrm{pH}$ 值, 从而引起氧化还原电位的变化.

\section{5 溶液 $\mathrm{pH}$ 值对光催化反应效率的影响}

乙醇胺溶液浓度为 $0.05 \mathrm{~mol} \cdot \mathrm{L}^{-1}$, 用 $\mathrm{NaOH}$ 和 $\mathrm{HCl}$ 溶液调节溶液 $\mathrm{pH}$ 值，在相同的反应条件下进 行研究, 发现溶液 $\mathrm{pH}$ 值对光催化反应产氢效率有 显著影响，如图 4 所示. 反应最佳的 $\mathrm{pH}$ 值范围为 $4 \sim 10,10<\mathrm{pH}<4$ 条件下产氢效率都相对较低. 乙醇胺溶液呈现出碱性 未经调节的 $0.05 \mathrm{~mol} \cdot \mathrm{L}^{-1}$ 乙醇胺水溶液 $\mathrm{pH}=10.70$ ), 而且碱性会随着乙醇 胺溶液浓度的增加而增强，这与前文提到的高浓度 区域乙醇胺浓度继续增加时反应效率明显下降这 一实验事实是一致的.

光催化反应效率受溶液 $\mathrm{pH}$ 值影响的原因是: 在 不同 $\mathrm{pH}$ 值的水溶液中, $\mathrm{TiO}_{2}$ 催化剂表面的电荷分 布状态不同，并呈现出不同的电负性，水溶液中的 $\mathrm{TiO}_{2}$ 表面羟基的质子与去质子化存在下列的平衡:

$-\mathrm{Ti}-\mathrm{OH}_{2}{ }^{+} \longleftrightarrow \mathrm{Ti}-\mathrm{OH}+\mathrm{H}^{+} \longleftrightarrow \mathrm{Ti}-\mathrm{O}^{-}+2 \mathrm{H}^{+}$ 水溶液中的乙醇胺也存在下列的平衡:
$\mathrm{HOCH}_{2} \mathrm{CH}_{2} \mathrm{NH}_{2}+2 \mathrm{H}_{2} \mathrm{O} \longleftrightarrow \mathrm{HOCH}_{2} \mathrm{CH}_{2} \mathrm{OH}+$

$\mathrm{NH}_{4}{ }^{+}+\mathrm{OH}^{-} \longleftrightarrow \mathrm{NH}_{2} \mathrm{CH}_{2} \mathrm{CH}_{2} \mathrm{O}^{-}+\mathrm{H}_{3} \mathrm{O}+\mathrm{H}_{2} \mathrm{O}$

在酸性中, 半导体 $\mathrm{Ti}-\mathrm{O}^{-}$表面与乙醇胺 呈电 负性, $\mathrm{NH}_{2} \mathrm{CH}_{2} \mathrm{CH}_{2} \mathrm{O}^{-}$) 存在电荷排斥作用而不利于 其在催化剂表面的有效吸附，在碱性中， $\mathrm{Ti}-\mathrm{OH}_{2}^{+}$ 表面与乙醇胺 (呈电中性) 存在电荷相吸作用, 有利 于乙醇胺在催化剂表面的有效吸附，也有利光催化 反应的进行，但当乙醇胺浓度很大或碱性较强时, $\mathrm{NH}_{4}^{+}$及 $\mathrm{OH}^{-}$会改变催化剂表面化学成分，引起催 化剂失活。溶液 $\mathrm{pH}$ 值的不同也会改变乙醇胺的氧 化还原电位. 这与我们以前研究低碳醇光催化制 氢反应规律相似 ${ }^{[9-10]}$.

\section{6 乙醇胺氧化重整中间物种验证}

Satoshi 等人 ${ }^{[8]}$ 对乙醇胺、二乙醇胺和三乙醇胺 的光催化氧化反应机理进行过探讨, 发现气相产物 为 $\mathrm{CO}_{2}$, 液相中产物为铵离子 $\left(\mathrm{NH}_{4}^{+}\right)$和硝酸根离子 $\left(\mathrm{NO}_{3}^{-}\right)$.

为定性证实上述推测的合理性，我们对反应 中间物种进行了 H-NMR 的研究, HNMR 图谱如 图 5 所示.从图中可以看出, 在光照 $5 \mathrm{~d}$ 后, 反应产 物谱图中出现了明显的变化, 反应体系中出现了氨 基取代的羰基类 $\left.\mathrm{H}_{2} \mathrm{~N}-\mathrm{CO}^{-}\right)$物质, 由于浓度较低和 分子内氢键相互作用, 所以也可以看到氨基中的 $\mathrm{H}$, 只是强度较小。说明主要反应物是 $\mathrm{HOCH}_{2} \mathrm{CH}_{2} \mathrm{NH}_{2}$, 当然不排除水解产物 $\mathrm{HOCH}_{2} \mathrm{CH}_{2} \mathrm{OH}$ 也参与氧化反 应. 乙醇胺分子重整的步骤可能经历了羟基化过 程，并进一步反应生成羰基物种的过程，这个过程 不断重复进行，最后经过脱羰过程生成 $\mathrm{CO}_{2}$ 和 $\mathrm{H}_{2} \mathrm{O}$, 同时水中的质子还原为氢, 这一过程相当复 杂, 更进一步的机理研究正在进行之中.

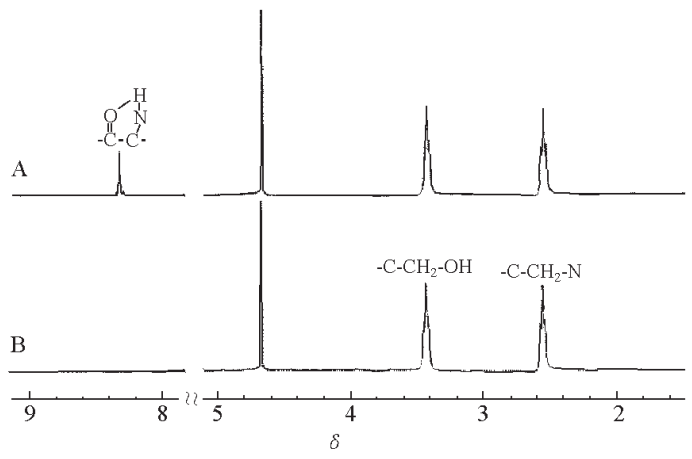

图 $5 \mathrm{Pt} / \mathrm{TiO}_{2}$ 光催化降解乙醇胺反应前后的 HNMR 谱图

Fig. 5 HNMR spectra of products of photocatalytic reforming degradation ethanolamine over $\mathrm{Pt} / \mathrm{TiO}_{2}$ photocatalyst

A) after photocatalytic reaction; B)before photocatalytic reaction 


\section{3 结 论}

(1) $\mathrm{Pt}$ 担载的 $\mathrm{TiO}_{2}$ 光催化剂能够有效地光催 化重整乙醇胺溶液，使其降解的同时制得氢，实现 了利用光催化方法同时消除有机污染物和制取氢 气两个目标。产生氢气的体积与光照时间有很好的 线性关系，产氢速率与乙醇溶液反应速率相近，要 比光催化分解纯水的反应速率高很多.

（2）乙醇胺浓度的影响较为复杂，浓度变化会 引起光催化剂表面吸附层浓度的变化和体系 $\mathrm{pH}$ 值 的变化,共同影响着光催化效率。

（3）光催化重整降解乙醇胺制氢的反应酸碱度 条件比较温和, 酸或碱性条件下产氢效率都相对较 低, 最佳的 $\mathrm{pH}$ 值范围为 $4 \sim 10$.

\section{References \\ $1 \mathrm{Hu}, \mathrm{Y} . \mathrm{M}$. Tech-Economics in Petrochemicals, 2000, 16 (5): 37 [胡玉梅。石油化工技术经济 (Shiyou Huangong Jishu Jinji), 2000, 16(5): 37 ] \\ 2 Ning, S. J.; Wei, D. Z. Plant Physiology Communications,}

2000, 36 (3): 219 [宁书菊, 魏道智。植物生理学通讯 (Zhiwu Shenglixue Tongxun), 2000, 36(3): 219]

$3 \mathrm{Niu}, \mathrm{K} . \mathrm{Y} . ; \mathrm{Hu}, \mathrm{Y} . \mathrm{Y}$. Chemical Industry and Labor Protection, 2000，21(5)：175 [牛魁尧, 胡颖颖. 化工劳动保护 (Huagong Laodong Baohu), 2000, 21(5): 175]

4 Paola, A. D. ; Garcia-López, E. ; Ikedab, S. Catalysis Today, 2002, 75: 87

5 Hamill, N. A. ; Weatherley, L. R.; Hardacre, C. Applied Catalysis B: Environmental, 2001, 30: 49

6 Piscopo, A.; Robert, D. ; Weber, J. V. Applied Catalysis B: Environmental, 2001, 35: 117

7 Curcó, D. ; Giménez, J. ; Addardak, A. Catalysis Today, 2002, 76: 177

8 Satoshi, H.; Natsuko, W.; Miki, M.; Hisao, H.; Nick, S. New J. Chem., 2001, (8): 999

9 Wu, Y. Q.; Lü, G. X.; Zhou, Q. ; Jiao, S. J. J. Molecular Catalysis, 2002，16: 101 [吴玉琪,吕功煊, 周 全,焦述俊. 分子催化 (Fengzi Cuihua)，2002，16: 101]

10 Wu, Y. Q. ; Lü, G. X. J. Molecular Catalysis, 2001, 15: 467 [吴玉琪, 吕功煊．分子催化 (Fengzi Cuihua), 2001，15: 467]

11 Hermann, J. M. ; Disdier, J.; Pichat, P. J. Phys. Chem., 1986, 90: 6028

12 Wu, Y. Q.; Lü, G. X. J. Molecular Catalysis, 2004, 18(2): 125 [吴玉琪, 吕功煊. 分子催化 (Fengzi Cuihua), 2004, 18 (2): 125]

\title{
Hydrogen Production by $\mathrm{Pt} / \mathrm{TiO}_{2}$ Anaerobic Photocatalytic Reforming Degradation of Aqueous Monoethanolamine*
}

\author{
Wu Yu-Qi Lü Gong-Xuan Li Shu-Ben
}

( State Key Laboratory for Oxo Synthesis and Selective Oxidation, Lanzhou Institute of Chemical Physics, Chinese Academy of Sciences, Lanzhou 730000$)$

\begin{abstract}
The production of hydrogen by anaerobic photocatalytic reforming degradation of aqueous monoethanolamine (ethanolamine, shortened form in the following text) solution has been investigated using a Pt/ $\mathrm{TiO}_{2}$ catalyst. The effect of many factors such as ethanolamine concentration, catalyst surface chemical state, and $\mathrm{pH}$ of systems on the photocatalytic production of hydrogen have been studied in detail. The catalyst and the products were characterized using HNMR XRD and XPS techniques, the possible reaction mechanism was discussed. The hydrogen production was achieved simultaneously with the photocatalytic reforming degradation of aqueous ethanolamine solution. It was showed that the aim of both producing hydrogen and photocatalytic reforming degradation of aqueous organic pollutant could be achieved using a $\mathrm{Pt} / \mathrm{TiO}_{2}$ catalyst. The $\mathrm{Pt}$ particles were identified as existed in $\mathrm{Pt}^{0}$ state on the $\mathrm{TiO}_{2}$. The $\mathrm{pH}$ and the ethanolamine concentration affect the hydrogen production rate significantly. At the same time, it was found that the optimum concentration of ethanolamine, $\mathrm{pH}$ value and Pt loading is $0.05 \mathrm{~mol} \cdot \mathrm{L}^{-1}, 4 \sim 10$ and $0.5 \% \sim 1.0 \%$ respectively. And the amino substituted carbonyl compounds was the main intermediates.
\end{abstract}

Keywords: Photocatalytic reforming, Degradation, Monoethanolamine, $\mathrm{Pt} / \mathrm{TiO}_{2}, \quad \mathrm{H}_{2}$ production

Received: December 16, 2003; Revised: February 16, 2004. Correspondent: Lü Gong-Xuan(E-mail: gxlu@ @ns. lzb. ac. cn; Tel: 0931-4968178). *The Project Supported by NKBRSF(2003CB214500), NSFC(90210027) and NKBRP 